\title{
Mass spectrometry analysis of rat cerebrospinal fluid proteins following exposure to the neurotoxicant carbonyl sulfide
}

\author{
O. Lardinois ${ }^{1}$, P.J. Kirby ${ }^{2}$, D. L. Morgan ${ }^{2}$, R.C. Sills ${ }^{3}$, K.B. Tomer ${ }^{1}$, and L.J. Deterding ${ }^{1}{ }^{,}{ }^{*}$ \\ ${ }^{1}$ Laboratory of Structural Biology, National Institute of Environmental Health Sciences, NIH/ \\ DHHS, 111 T.W. Alexander Drive, PO Box 12233, Research Triangle Park, NC 27709 \\ ${ }^{2}$ Laboratory of Toxicology and Pharmacology, National Institute of Environmental Health \\ Sciences, NIH/DHHS, 111 T.W. Alexander Drive, PO Box 12233, Research Triangle Park, NC \\ 27709 \\ ${ }^{3}$ Cellular and Molecular Pathology, National Institute of Environmental Health Sciences, NIH/ \\ DHHS, 111 T.W. Alexander Drive, PO Box 12233, Research Triangle Park, NC 27709
}

\begin{abstract}
RATIONALE-Using a proteomic-based approach we have investigated possible altered expression of a range of cerebral spinal fluid (CSF) proteins following exposure to the neurotoxicant carbonyl sulfide (COS). CSF is ideal for the investigation of markers of brain injury or disease since it is secreted from several central nervous system structures and changes in the CSF composition may reflect brain insult and many pathological processes.
\end{abstract}

METHODS-Animals were placed in exposure chambers and were exposed to $0 \mathrm{ppm}$ or $500 \mathrm{ppm}$ carbonyl sulfide for one, two or three days, six hours per day. After the last inhalation exposure, 50-70 $\mu \mathrm{L}$ CSF sample was obtained by lumbar puncture. CSF samples were analyzed by ESI/MS on either a Q-TOF Premier or an Agilent 6340 ion trap and by MALDI/MS on a 4800 MALDITOF/TOF Analyzer.

RESULTS-The dynamic range of abundance of the identified proteins spanned over more than three orders of magnitude. The four most abundant proteins identified (albumin, cystatin C, serotransferrin, transthyretin) are major proteins that are present in both CSF and blood at high levels but the fifth most abundant protein identified (prostaglandin H2D isomerase) is the second most abundant protein in human CSF and is secreted and synthesized in rat central nervous system. No significant differences were observed between carbonyl sulfide treated CSF samples and the control CSF samples because of blood contamination.

CONCLUSIONS-Quantitative MS protein analyses of rat CSF is limited by the low sample volumes that can practicably be obtained from rats and the low protein concentrations in rat CSF. Results of this work suggest a clear need of CSF collection that would minimize blood contamination.

*Corresponding author: Dr. Leesa J. Deterding, Laboratory of Structural Biology, NIH/National Institute of Environmental Health Sciences, 111 T.W. Alexander Drive, Research Triangle Park, NC 27709, USA, Phone: +1 (919) 541-3009, Fax: +1 (919) 541-0220, deterdi2@niehs.nih.gov. 


\section{Keywords}

mass spectrometry; carbonyl sulfide; cerebral spinal fluid

\section{Introduction}

Mass spectrometry (MS) has radically changed the biological sciences since the development of two soft ionization techniques for the analysis of biological macromolecules, matrix-assisted laser desorption ionization (MALDI) and electrospray ionization (ESI), both of which are used in the discipline of proteomics. Proteomics is the study of proteins and is crucial in understanding and combating disease through the identification of proteins, discovering disease biomarkers, studying protein involvement in specific metabolic pathways, and identifying protein targets in drug discovery. In general, discovery-based proteomic efforts lend themselves to global analyses whereby a broad survey of the proteome is performed across various samples. Because cerebrospinal fluid (CSF) is secreted from several central nervous system structures, changes in the CSF composition may reflect brain insult and many pathological processes. It is, thus, an ideal bodily fluid to investigate for markers of brain injury or disease. Proteomics has been used to investigate the proteins in cerebrospinal fluid [1-5]. Potential biomarkers of some common neurodegenerative diseases may help in the diagnosis, treatment and/or monitoring of an identified disease [6-15].

In this study, we use proteomics in an effort to identify markers of neurological disease following environmental exposure to carbonyl sulfide (COS) in rats [16-18]. We focused on carbonyl sulfide which is a low molecular weight, colorless gas that originates from a variety of sources. Carbonyl sulfide is the most abundant sulfur compound naturally present in the atmosphere because it is emitted from oceans, volcanoes and deep sea vents. The largest man-made sources of carbonyl sulfide release include its primary use as a chemical intermediate and as a byproduct of carbon disulfide production; however, it is also released from a number of sources including automobiles and coal-fired power plants. The average total worldwide release of carbonyl sulfide to the atmosphere has been estimated at about 3 million tons/year, of which less than one third was related to human activity [19]. Carbonyl sulfide can also be found in some food, such as cheese and prepared vegetables of the cabbage family.

In the rat model, exposure to $500 \mathrm{ppm} \operatorname{COS}$ for six hours per day for two days resulted in no microscopic lesions in the brain. By the third day of COS exposure, bilateral symmetrical necrosis was present in the posterior colliculus of all rats exposed to $500 \mathrm{ppm}[16,18]$. The time points of 1,2 and 3 days of exposure to $500 \mathrm{ppm}$ COS were selected to examine possible predictors/biomarkers of neuronal necrosis and inflammation in the CSF. Because of the relative lack of toxicity data and persistence of the gas in the atmosphere, COS is listed as a high priority Clean Air Act chemical by the United States Environmental Protection Agency. Because cerebrospinal fluid circulates within the ventricles of the brain where it plays an essential physiological role in homeostasis of neuronal cells, CSF was considered a good biological sample for measuring biomarkers that are linked to carbonyl 
sulfide neurotoxicity. In this study, we use proteomics to identify markers of neurological disease following COS exposure.

\section{EXPERIMENTAL}

\section{Chemicals and Reagents}

Protease inhibitors (aprotinin and leupeptin), dithiothreitol, and iodoacetamide were all purchased from Sigma-Aldrich Corporation (St. Louis, MO). Sequencing grade porcine trypsin was purchased from Promega Corporation (Madison, WI). Solvents for liquid chromatography were purchased as follows: HPLC-grade acetonitrile (Fisher Scientific, Fairlawn, NJ) and formic acid (Sigma-Aldrich Corporation, St. Louis, MO). Yeast alcohol dehydrogenase (ADH) digest used for mass spectrometric quantification of proteins was obtained from Waters Corporation (Milford, MA).

\section{Inhalation Exposure and CSF Sample Collection}

Ninety Fisher male 344 rats (8-9 weeks old) were used. Animals were placed in exposure chambers for two days before chemical exposure for acclimation to exposure conditions. They were exposed to $0 \mathrm{ppm}$ (controls) or $500 \mathrm{ppm}$ COS for one, two or three days, six hours per day. The concentrations of COS in the exposure chambers were verified by gas chromatography. After the last inhalation exposure, rats were sacrificed and 50-70 $\mu$ L CSF sample was obtained by lumbar puncture. The samples were immediately stored at $-80{ }^{\circ} \mathrm{C}$ until tested.

\section{ELISA}

The degree of blood contamination in the CSF samples was assessed by ELISA using a rabbit anti-rat hemoglobin polyclonal antibody (AbD Serotec, Kidlington, UK). Detection of antigen-antibody complexes was performed using standard 'indirect' ELISAs (no capture antibody). Aliquots of CSF samples (1:12,500) in coating buffer (100 mM sodium bicarbonate, $\mathrm{pH}$ 9.6) were transferred to 96 -well plates (Greiner Labortechnik, Frickenhausen, Germany) and the plates were incubated for $60 \mathrm{~min}$ at $37^{\circ} \mathrm{C}$. Plate wells were then washed once with $1 \times$ TBS washing buffer $(0.05 \%$ Tween $20,0.2 \%$ cold-water fish skin gelatin, $\mathrm{pH}$ 7.4) and blocked with coating buffer ( $100 \mathrm{mM}$ sodium bicarbonate, $\mathrm{pH}$ 9.6, containing $4 \%$ cold-water fish skin gelatin) for $60 \mathrm{~min}$ at $37^{\circ} \mathrm{C}$. Thereafter, the rabbit anti-rat hemoglobin serum (1:5000) in washing buffer was added and incubated at $37{ }^{\circ} \mathrm{C}$ for $60 \mathrm{~min}$. After three washes, the secondary antibody, anti-rabbit IgG-alkaline phosphatase (Pierce Chemical Co., Rockford, IL), 1:5000 in washing buffer, was added and incubated for $60 \mathrm{~min}$ at $37{ }^{\circ} \mathrm{C}$. After three additional washes, the antigen-antibody complexes were developed using a CDP-Star chemiluminescence system (Roche Molecular Biochemicals, Indianapolis, IN) and the chemiluminescence signal was detected using a Tecan SpectraFluor Plus microplate reader (Tecan US, Research Triangle Park, NC) and processed using the Xfluor ${ }^{\mathrm{TM}}$ software (Tecan US).

\section{Bradford Assays}

Protein concentrations were determined by the Bradford assay using a BSA standard curve. 


\section{Fractionation of CSF}

CSF samples were fractionated according to Zhang and coworkers [20] using acetonitrile. CSF samples were mixed with 1.5 volumes of acetonitrile at room temperature for $30 \mathrm{~min}$. The pellet (Pellet 1; P1) was collected by centrifugation at 15,000 $\times \mathrm{g}$ at room temperature. The supernatant (Supernatant $1 ; \mathrm{S} 1$ ) was incubated with 3 volumes of acetonitrile at room temperature for $30 \mathrm{~min}$, and then centrifugated as above to produce the second pellet (Pellet $2 ; \mathrm{P} 2$ ) and supernatant (Supernatant 2; S2). The three fractions were lyophilized and stored at $-80{ }^{\circ} \mathrm{C}$.

\section{Enzymatic digestion}

Following fractionation and lyophilization, the samples were redissolved in $100 \mathrm{mM}$ ammonium bicarbonate buffer, $\mathrm{pH} 8.0$, reduced and alkylated, and digested overnight at 37 ${ }^{\circ} \mathrm{C}$ with trypsin. The digests were then stored at $-80{ }^{\circ} \mathrm{C}$ for subsequent MS analysis.

\section{Mass Spectrometry}

MALDI Analyses-Prior to MALDI/MS analyses, porcine renin substrate tetradecapeptide, an internal standard for quantification, was spiked at different concentration levels into the digested CSF samples. The resulting mixtures were desalted and purified by ZipTip C18 pipette tips (Millipore, Bedford, MA), following the procedure described in the ZipTip user's guide. All MALDI/MS analyses were performed on a 4800 MALDI-TOF/TOF Analyzer (AB Sciex, Foster City, CA) operated in the positive ion reflector mode. Samples were deposited on a stainless steel MALDI target plate using a standard fast evaporation protocol that included spotting $0.5 \mu \mathrm{L}$ of MALDI matrix onto the target plate, applying a $0.5 \mu \mathrm{L}$ aliquot of ZipTip C18-purified peptide samples on top of the matrix bed, and air drying of samples before collection of MS and MS/MS spectra. A saturated solution of a-cyano-4-hydroxycinnamic acid in 45:45:10 ethanol/water/formic acid (v/v) was used as the MALDI matrix. Spectra were initially externally calibrated using a protein calibration mixture (Bruker Daltonics, Bremen, Germany). In the second phase of the calibration process, trypsin autolysis peaks were used as an internal calibrant. The data were processed and analyzed by a Global Protein Server Workstation (AB Sciex) using Mascot software (Matrix Science, London, UK) to search peptide mass fingerprints and MS/MS data. Data obtained by MALDI/MS were also processed using MarkerView ${ }^{\mathrm{TM}}$ software (Applied Biosystems/MDS Sciex, Toronto, Canada). MarkerView ${ }^{\mathrm{TM}}$ software is a novel program designed for rapid review of data. Principal component analysis (PCA) was performed on processed data using different scaling algorithms (Mean centering, Autoscaling, and Pareto scaling) as proposed by the MarkerView ${ }^{\mathrm{TM}}$ statistical software program.

ESI Analyses-Data were acquired either on a Q-TOF or an ion trap mass spectrometer. A Waters Q-TOF Premier mass spectrometer equipped with a nanoAcquity UPLC system and nanoLockspray source (Waters, Milford, MA) was used. For these LC/MS analyses, the digests were injected directly onto the LC column, and separations were performed using a nanoAcquity Atlantis C18 reversed-phase column $(100 \mu \mathrm{m} \times 100 \mathrm{~mm}, 3 \mu \mathrm{m}$ particle size $)$ at a flow rate of $300 \mathrm{~nL} / \mathrm{min}$. A Symmetry C18 trapping column $(180 \mu \mathrm{m} \times 20 \mathrm{~mm}, 5 \mu \mathrm{m}$ 
particle size) was positioned in-line with the analytical column. Typically, a $5 \mu \mathrm{L}$ aliquot of the tryptic digest spiked with $50 \mathrm{fmol}$ of MassPREPTM yeast alcohol dehydrogenase digestion standard (Waters Corporation) was trapped onto the column, and peptides were eluted using a linear gradient of $98 \%$ solvent $\mathrm{A}(0.1 \%$ formic acid in water $(\mathrm{v} / \mathrm{v}))$ and $2 \%$ solvent B $(0.1 \%$ formic acid in acetonitrile $(\mathrm{v} / \mathrm{v}))$ to $40 \%$ solvent B over $90 \mathrm{~min}$. The lockmass calibrant peptide standard, $500 \mathrm{fmol} / \mu \mathrm{L}$ [Glu 1]-fibrinopeptide B human, was infused into the nanoLockSpray ion source at a flow rate of $500 \mathrm{~nL} / \mathrm{min}$ and was sampled during the acquisition at $30 \mathrm{~s}$ intervals. The instrument was operated in $\mathrm{V}$-mode using the ' $M S^{\mathrm{e}}$ ' mode of acquisition with the collision cell operating with two alternating collision energies, $5 \mathrm{eV}$ for the low collision energy range and ramping from 20 to $35 \mathrm{eV}$ for the high collision energy range. The low energy scans provide information about intact peptides, whereas high energy scans result in fragmentation of polypeptides. The data were processed using ProteinLynx Global Server (PLGS) software (Waters Corporation).

Ion trap analyses were performed on an Agilent 1100 Series HPLC-Chip/MS (Agilent Technologies, Santa Clara, CA) interfaced to an Agilent 6340 Ion Trap. The HPLC-Chip configuration consisted of a $40 \mathrm{~nL}$ enrichment column and a $43 \mathrm{~mm} \times 75 \mu \mathrm{m}$ analytical column (ZORBAX 300SB C18). A $10 \mu \mathrm{L}$ aliquot of Zip-Tip C18-purified peptide samples was loaded onto the chip in each LC/MS analysis. Linear gradients of 5-50\% solvent B were performed over $45 \mathrm{~min}$ at a flow rate of $500 \mathrm{~nL} / \mathrm{min}$. Parameter settings for positive ion ESIMS were as follows: capillary voltage, $-2000 \mathrm{~V}$; end plate offset, $500 \mathrm{~V}$; capillary exit, 180 $\mathrm{V}$; dry gas, $4 \mathrm{~L} / \mathrm{min}$; dry gas temperature, $325^{\circ} \mathrm{C}$. For MS/MS, automated data-dependent acquisitions of the four most abundant ions were employed with a fragmentation amplitude of $0.80 \mathrm{~V}$, which was scanned from $30 \%$ to $200 \%$ of this preset value (SmartFrag parameter on the instrument tune page). Automated database searching was performed using the data extractor feature of the SpectrumMill software from Agilent. The resulting extracted data were then searched against the NCBI non-redundant data base using the MS/MS Search function in the SpectrumMill software.

\section{RESULTS AND DISCUSSION}

\section{Blood Contamination}

The CSF samples were first qualitatively evaluated for blood contamination by visual inspection. The result of this analysis is presented in Table 1. CSF is a colorless fluid, thus, samples having a red color $(n=39)$ were considered as heavily contaminated by blood and were not used. The most abundant protein in whole blood, hemoglobin, is a useful marker of blood contamination [21]. The degree of blood contamination in the remaining colorless samples was therefore quantitatively assessed by ELISA using an anti-rat hemoglobin antibody. CSF with hemoglobin levels higher than 1/500,000 dilution of a whole blood sample can be considered contaminated by blood [22]. Based on the ELISA assay the majority of the CSF samples that were used were considered contaminated (44 of 51 samples with at least 1/500 000 blood). CSF samples were also analyzed for total protein content. Figure 1 shows that blood contamination, assessed by ELISA using the anti-rat hemoglobin antibody, has an impact on the total amount of protein in the CSF samples. The 
figure clearly demonstrates a direct proportionality between the level of contamination by blood and the overall total protein concentration in the samples.

The direct lumbar puncture protocol to collect CSF in rats used in the present study was first developed by De la Calle and Paíno [23]. A refinement of this protocol that minimizes blood contamination was later developed by Wang et al. [24]. Using this procedure, the authors reported a significant decrease in visible blood contamination (from $24.9 \%$ to $11.0 \%$ ). Besides lumbar puncture, the most commonly used methods require the implantation of cannulas or catheters with or without dialysis membrane into the rat brain [25-28]. These approaches sample CSF from the cisterna magna, the largest CSF compartment lying between the cerebellum and the dorsal surface of the medulla oblongata. Implanted catheters do offer unquestionable advantages but their implantation is very invasive, time-consuming and the surgery needed is complicated. In experiments where large numbers of rats are used, the use of direct lumbar puncture is therefore advantageous. Unfortunately, blood contamination of the CSF is common for all of these established sampling techniques. Visible blood contamination of the CSF samples are often 20-30\% or higher [23, 26-28]. In addition, a clear CSF sample upon macroscopic inspection does not necessary mean that it is not contaminated by blood and additional methods are needed to further control for blood contamination. In the present study, we have quantified the level of hemoglobin in CSF as an additional index for blood contamination as originally described by Zhang [22]. This approach is particularly helpful for samples that have been previously frozen, where red blood cell counts are not available.

\section{Fractionation of CSF}

Representative MALDI mass spectra of a CSF sample which was fractionated using acetonitrile are presented in Figure 2. The ions labeled in green, red and blue correspond in mass to tryptic peptides from albumin, hemoglobin and cystatin, respectively. The results demonstrate that the majority of albumin $(>90 \%)$ was precipitated in the pellet one fraction. In addition, some lower abundance proteins were significantly enriched in pellet two.

\section{Quantification of proteins in rat CSF}

In the initial phase of this project, a small set of CSF samples $(n=10)$ from control animals were pooled, and this naïve CSF sample was characterized by LC/MS/MS on an ion trap MS using DDA and on a Q-TOF MS using MS ${ }^{\mathrm{e}}$. A total of ca. 80 proteins were identified of which 60 could be quantified using $\mathrm{MS}^{\mathrm{e}}$. The dynamic range of abundance of the identified proteins spanned over more than three orders of magnitude (Figure 3). The four most abundant proteins identified (albumin, cystatin C, serotransferrin, and transthyretin) are major proteins that are present in both the CSF and blood at high levels [29]. However, it has been shown that the ratios of the concentration of both cystatin $\mathrm{C}$ and transthyretin are significantly greater in CSF than in blood plasma [30]. The fifth most abundant protein identified, prostaglandin $\mathrm{H} 2 \mathrm{D}$ isomerase, is the second most abundant protein in human CSF after albumin [31]. This protein is secreted and synthesized in rat central nervous system by astrocytes [32] but has also been detected in various biological fluids including serum [32]. The data also show the presence of hemoglobin at 600-900 nM/L levels. As hemoglobin is blood-specific, this would lead one to expect that the quantitation of many proteins in Figure 
3 may have been altered as a result of the contamination of the naïve CSF sample by blood. Next, CSF samples with less than 1/500,000 blood contamination from untreated controls and COS-treated samples were analyzed by LC/ESI/MS/MS for protein quantification. These analyses were hampered, however, by the limited amount of proteins in the sample which typically were below the limit of quantification.

\section{Principal Component Analysis}

Because MALDI/MS generally requires less material than ESI/MS, the individual proteolytic sample digests were re-analyzed using MALDI TOF/TOF (Table 2). Results indicate that MALDI/MS is sensitive enough to quantify the most abundant proteins in the samples. Statistical comparisons between untreated controls and treated samples were performed by principal component analysis using the MarkerView ${ }^{\mathrm{TM}}$ software. This software provides both supervised (PCA-DA) and unsupervised (PCA) methods and both produce two plots: i) the scores plot displays the natural clustering or separation within the data set, and ii) the loadings plot identifies those variables that contribute most significantly to the variance observed within the data set. Correlated variables in the loadings plot lie on straight lines that pass through the origin. The variables in the loadings plot that contribute the most to the variance are those furthest from the origin of the plot. The score and loading plots of CSF samples are presented in Figures 4 to 7. The PCA scores plot (shown in Figure 4) was obtained without input of any prior knowledge of the sample groups and indicates that the CSF samples can clearly be separated on the basis of the contamination by blood (red indicates high contamination, green indicates low contamination). The PCA-DA scores plot in Figure 5 uses prior knowledge of the sample group (in this case, the amount of hemoglobin contamination) to identify the variable that contributes most significantly to the difference. From the loading plots shown in Figure 4 and 5 from 25 control animals and 26 treated animals, the ions that contribute most significantly to the variance within the data set are determined to be the ions of $\mathrm{m} / \mathrm{z} 1013.51,1274.73,1572.74,2195.12$ and 2567.30. These ions correspond in mass to tryptic peptides T5a, T4 $\beta$, T4 $\alpha$, T2-3 $\beta$ and T4-5 a from rat hemoglobin, respectively, thereby indicating that the significant variability observed between the samples can be explained by blood contamination. Similar statistical analyses performed by grouping the samples on the basis of COS-exposure reveals no significant differences between COS-treated CSF samples and the control CSF samples (Figures 6 and $7)$.

In regards to identifying predictors/biomarkers of COS neurotoxicity, additional factors may have contributed to the findings in this study. First, the CSF was collected from the lumbar CSF which may not be as representative as CSF from the brain for detecting biomarkers and it is known that rostro-caudal gradients can have an effect on markers [33]. Second, the CSF was collected on day 3 of COS exposure when consistent areas of necrosis were seen in the posterior colliculus. The necrosis in the posterior colliculus was focal and may not have been extensive enough for identifying biomarkers of inflammation and necrosis in the CSF. By day 4 , necrosis was detected in many sites of the brain including the frontoparietal cortex, putamen, retrosplenial cortex, hippocampus, thalamus, red nucleus, anterior olivary nucleus and nucleus of lateral lemniscus. Compared to the 3 days of COS exposure, it would be of interest to determine whether or not the 4 day time point would be sensitive for 
identifying predictive biomarkers of inflammation and necrosis in CSF samples which were not contaminated with blood.

\section{CONCLUSIONS}

Blood contamination is a critical factor to consider when assessing the quality of CSF samples. Minor contamination of CSF with blood may dramatically alter the protein profile when using MS determination. Other factors to consider when analyzing rat CSF are the low sample volumes that can practicably be obtained from rats $(\sim 50 \mu \mathrm{L})$ and the low protein concentrations in rat CSF $\left(\sim 1 / 200^{\text {th }}\right.$ to $\sim 1 / 400^{\text {th }}$ that of blood). CSF samples with less than $1 / 500,000$ blood contamination contained very limited amount of proteins which were below the limit of quantification of the ion trap and QTOF instruments used in this investigation. MALDI/MS was sensitive enough to allow relative quantification of the most abundant proteins in the sample, however, no significant differences were observed between COStreated CSF samples and the control CSF samples using principal component analysis (PCA) and MALDI/MS. Correct group discrimination and selection of specific markers (e.g. tryptic hemoglobin peptides T4a, T5a, T4 $\beta$, T2-3 $\beta$, and T4-5a) were achieved using both supervised (PCA) and unsupervised (PCA-DA) methods when comparing CSF samples contaminated by blood versus blood free-samples. Results of this work suggest a clear need to use an alternative method of CSF collection that would minimize blood contamination.

\section{Acknowledgments}

This research has been supported by the Intramural Research Program of the NIH, National Institute of Environmental Health Sciences. The authors would like to acknowledge Dr. Jeffrey Kuhn (NIEHS/NIH) and Dr. Jason Williams (NIEHS/NIH) for critical review of the manuscript, and the NIEHS Protein Microcharacterization Core Facility for helpful suggestions and assistance.

\section{Abbreviations}

LC-MS/MS liquid chromatography tandem mass spectrometry

CSF cerebral spinal fluid

COS carbonyl sulfide

\section{REFERENCES}

1. Yuan X, Desiderio DM. Proteomics analysis of human cerebrospinal fluid. J. Chromatography B. 2005; 815:179-189.

2. Hale JE, Gelfanova V, You JS, Knierman MD, Dean RA. Proteomics of Cerebrospinal Fluid: Methods for Sample Processing. Methods Mol. Biol. 2008; 425:53-66. [PubMed: 18369886]

3. Zougman A, Pilch B, Poetelejnikov A, Kiehntopf M, Schnabel C, Kumar C, Mann M. Integrated analysis of cerebrospinal fluid peptidome and proteome. J. Proteome Res. 2008; 7:286-399. [PubMed: 18078310]

4. Schutzer SE, Liu T, Natelson BJ, Angel TE, Schepmoes AA, Purvine SO, Hixson KK, Lipson JS, Camp DG II, Coyle PK, Smith RD, Bergquist J. Establishing the proteomic of normal human cerebrospinal fluid. PLOS One. 2010; 5:1-7.

5. Craft GE, Chen A, Nairn AE. Recent advances in quantitative neuroproteomics. Methods. 2013; 61:186-218. [PubMed: 23623823] 
6. D’Ascenzo M, Relkin NR, Lee KH. Alzheimer's disease cerebrospinal fluid biomarker discovery: A proteomics approach. Curr. Op. Mol. Therapeutics. 2005; 7:557-564.

7. Zhang J, Goodlett DR, Quinn JF, Peskind E, Kaye JA, Zhou Y, Pan C, Yi E, Eng J, Wang Q, Aebersold RH, Montine TJ. Quantitative proteomics of cerebrospinal fluid from patients with Alzheimer disease. J. Alzheimer's Disease. 2005; 7:125-133. [PubMed: 15851850]

8. Zhang J, Goodlett DR, Montine TJ. Proteomic discovery in cerebrospinal fluid for neurodegenerative diseases. J. Alzheimer's Disease. 2005; 8:377-386. [PubMed: 16556969]

9. Blennow K. CSF biomarkers for Alzheimer's disease: use in early diagnosis and evaluation of drug treatment. Exp. Rev. Mol. Diagn. 2005; 5:661-672.

10. Davidsson P, Sjögren M. Proteome studies of CSF in AD patients. Mechanisms of Ageing and Development. 2006; 127:133-137. [PubMed: 16293296]

11. Süssmuth SD, Brettschneider J, Ludolph AC, Tumani H. Biochemical markers in CSF of ALS patients. Current Medicinal Chemistry. 2008; 15:1788-1801. [PubMed: 18691039]

12. Brechlin P, Jahn O, Steinacker P, Cepek L, Kratzin H, Lehnert S, Jessee S, Mollenhauer B, Kretzschmar HA, Wiltfang J, Otto M. Cerebrospinal fluid-optimized two-dimensional difference gel electrophoresis (2-D DIGE) facilitates the differential diagnosis of Creutzfeldt-Jakob disease. Proteomics. 2008; 8:4357-4366. [PubMed: 18814332]

13. Sinha A, Srivastava N, Singh S, Singh AK, Bhurhan S, Shukla R, Singh JP. Identification of differentially displayed proteins in cerebrospinal fluid of Parkinson's disease patients: A proteomic approach. Clinica Chimia Acta. 2009; 400:14-20.

14. Zürbig P, Jahn H. Use of proteomic methods in the analysis of human body fluids in Alzheimer research. Electrophoresis. 2012; 33:3617-3630. [PubMed: 23160951]

15. Perrin RJ, Payton JE, Malone JP, Gilmore P, Davis AE, Xiong C, Fagan AM, Townsend RR, Hotzman DM. Quantitative label-free proteomics for discovery of biomarkers in cerebrospinal fluid: Assessment of technical and inter-individual variation. PLOS One. 2013; 8:1-17.

16. Morgan DL, Little PB, Herr DW, Moser VC, Collins B, Herbert R, Johnson GA, Maronpot RR, Harry GJ, Sills RC. Neurotoxicity of carbonyl sulfide in F344 rats following inhalation exposure for up to 12 weeks. Toxicol Appl Pharmacol. 2004; 200:131-145. [PubMed: 15476866]

17. Morrison JP, Ton TV, Collins JB, Switzer RC, Little PB, Morgan DL, Sills RC. Gene expression studies reveal that DNA damage, vascular perturbation, and inflammation contribute to the pathogenesis of carbonyl sulfide neurotoxicity. Toxicol. Pathol. 2009; 37:502-511. [PubMed: 19395590]

18. Sills RC, Morgan DL, Herr DW, Little PB, George NM, Ton TV, Love NE, Maronpot RR, Johnson GA. Contribution of magnetic resonance microscopy in the 12-week neurotoxicity evaluation of carbonyl sulfide in Fischer 344 rats. Tox. Path. 2004; 32:501.

19. Hazardous Substances Data Bank. National Library of Medicine; Carbonyl Sulfide CASRN: 463-58-1.

20. Zhang J, Goodlett DR, Peskind ER, Quinn JF, Zhou Y, Wang Q, Pan C, Yi E, Eng J, Aebersold RH, Montine TJ. Quantitative proteomic analysis of age-related changes in human cerebrospinal fluid. Neurobiol. Aging. 2005; 26:207-227. [PubMed: 15582749]

21. You JS, Gelfanova V, Knierman MD, Witzmann FA, Wang M, Hale JE. The impact of blood contamination on the proteome of cerebrospinal fluid. Proteomics. 2005; 5:290-296. [PubMed: 15672452]

22. Zhang J. Proteomics of human cerebrospinal fluid-the good, the bad, and the ugly. Proteomics Clin. Appl. 2007; 1:805-819. [PubMed: 21136735]

23. De la Calle J, Paíno C. A procedure for direct lumbar puncture in rats. Brain Res. Bull. 2002; 59:245-250. [PubMed: 12431755]

24. Wang X, Kimura S, Yazawa T, Endo N. Cerebrospinal fluid sampling by lumbar puncture in rats repeated measurements of nitric oxide metabolites. J. Neurosci. Methods. 2005; 145:89-95. [PubMed: 15922028]

25. Chou KJ, Donovan MD. Distribution of antihistamines into the CSF following intranasal delivery. Biopharm. Drug Dispos. 1997; 18:335-346. [PubMed: 9158881]

26. Consiglio AR, Lucion AB. Technique for collecting cerebrospinal fluid in the cisterna magna of non-anesthetized rats. Brain Res. Protoc. 2000; 5:109-114. 
27. Ylitalo P, Heikkinen ER, Myllylä VV. Evaluation of successive collections of cisternal cerebrospinal fluid in rats, rabbits, and cats. Exp. Neurol. 1976; 50:330-336. [PubMed: 1248554]

28. Sharma AK, Schultze AE, Cooper DM, Reams RY, Jordan WH, Snyder PW. Development of a percutaneous cerebrospinal fluid collection technique in F-344 rats and evaluation of cell counts and total protein concentrations. Toxicol Pathol. 2006; 34:393-395. [PubMed: 16844667]

29. Hühmer AH, Biringer RG, Amato H, Fonteh AN, Harrington MG. Protein analysis in human cerebrospinal fluid: Physiological aspects, current progress and future challenges. Dis. Markers. 2006; 22:3-26. [PubMed: 16410649]

30. Cole T, Dickson PW, Esnard F, Averill S, Risbridger GP, Gauthier F, Schreider G. The cDNA structure and expression analysis of the genes for the cysteine proteinase inhibitor cystatin $\mathrm{C}$ and $\beta$ 2-microglobulin in rat brain. Eur. J. Biochem. 1989; 186:35-42. [PubMed: 2689174]

31. Harrington MG, Aebersold R, Martin BM, Merrill CR, Hood L. Identification of a brain-specific human cerebrospinal fluid glycoprotein, beta-trace protein. Appl. Theor. Electrophor. 1993; 3:229-234. [PubMed: 7692978]

32. Giocomelli S, Leone MB, Grima J, Silvestini B, Cheng CY. Astrocytes synthesize and secrete prostaglandin D synthetase in vitro. Biochem. Biophys. Acta. 1996; 1310:269-276. [PubMed: 8599604]

33. Tarnaris A, Toma AK, Chapman MD, Petzold A, Keir G, Kitchen ND, Watkins LD. Rostrocaudal Dynamics of CSF. Biomarkers Neurochem. Res. 2011; 36:528-532. 


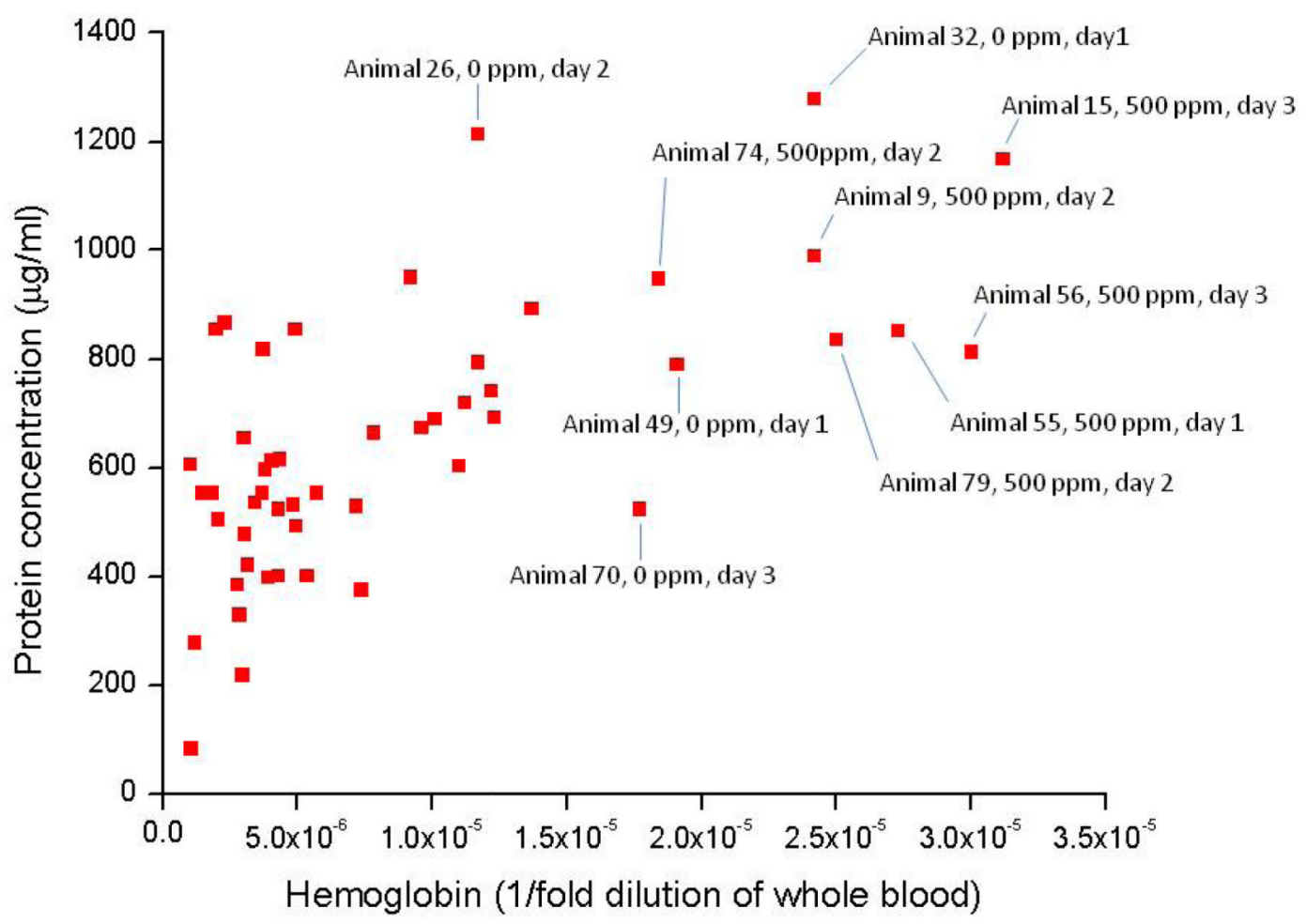

Figure 1.

Protein concentration versus hemoglobin levels in CSF samples. Blood contamination was quantitatively assessed by ELISA using an anti-rat hemoglobin antibody. The amount of protein in the CSF samples was measured using Bradford protein assay. 
hemoglobin

albumin

cystatin
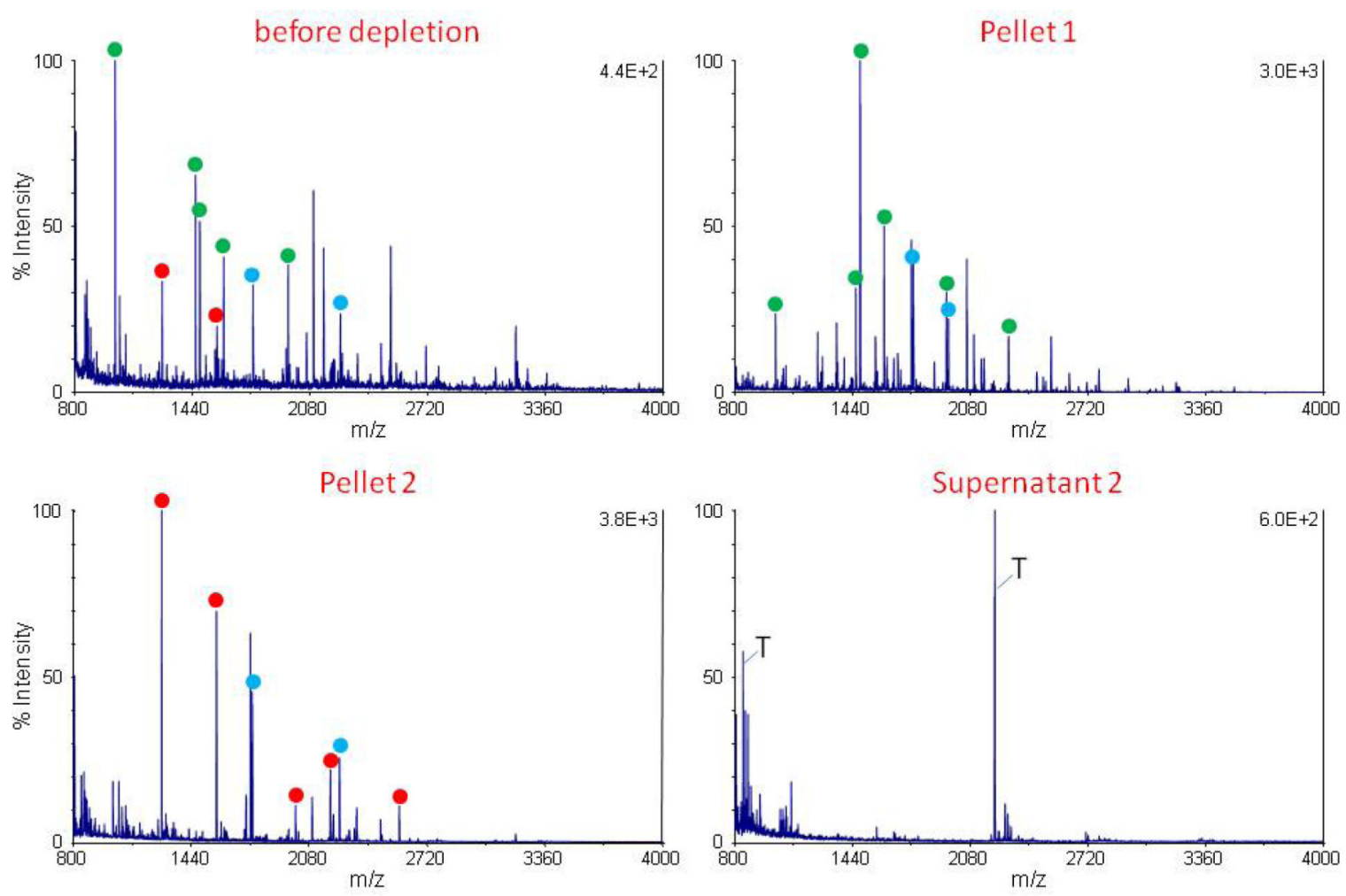

Figure 2.

Effect of fraction with acetonitrile precipitation on the CSF peptide profiles obtained by MALDI-TOF MS. A representative CSF sample with less than 1/500,000 blood contamination was incubated with 1.5 volumes and 3.0 volumes of acetonitrile, consecutively at room temperature to produce Pellet 1 (P1), Pellet 2 (P2) and Supernatant (S2) fractions. Aliquots of the CSF sample before precipitation and from P1, P2 and S2 fractions were digested with trypsin and the tryptic digests were analyzed by MALDI/MS. The ions at $\mathrm{m} / \mathrm{z} 842.50$ and 2211.10 (labeled T) correspond to the trypsin autodigestion products that were used as internal mass-calibration.

Rapid Commun Mass Spectrom. Author manuscript; available in PMC 2015 December 15. 


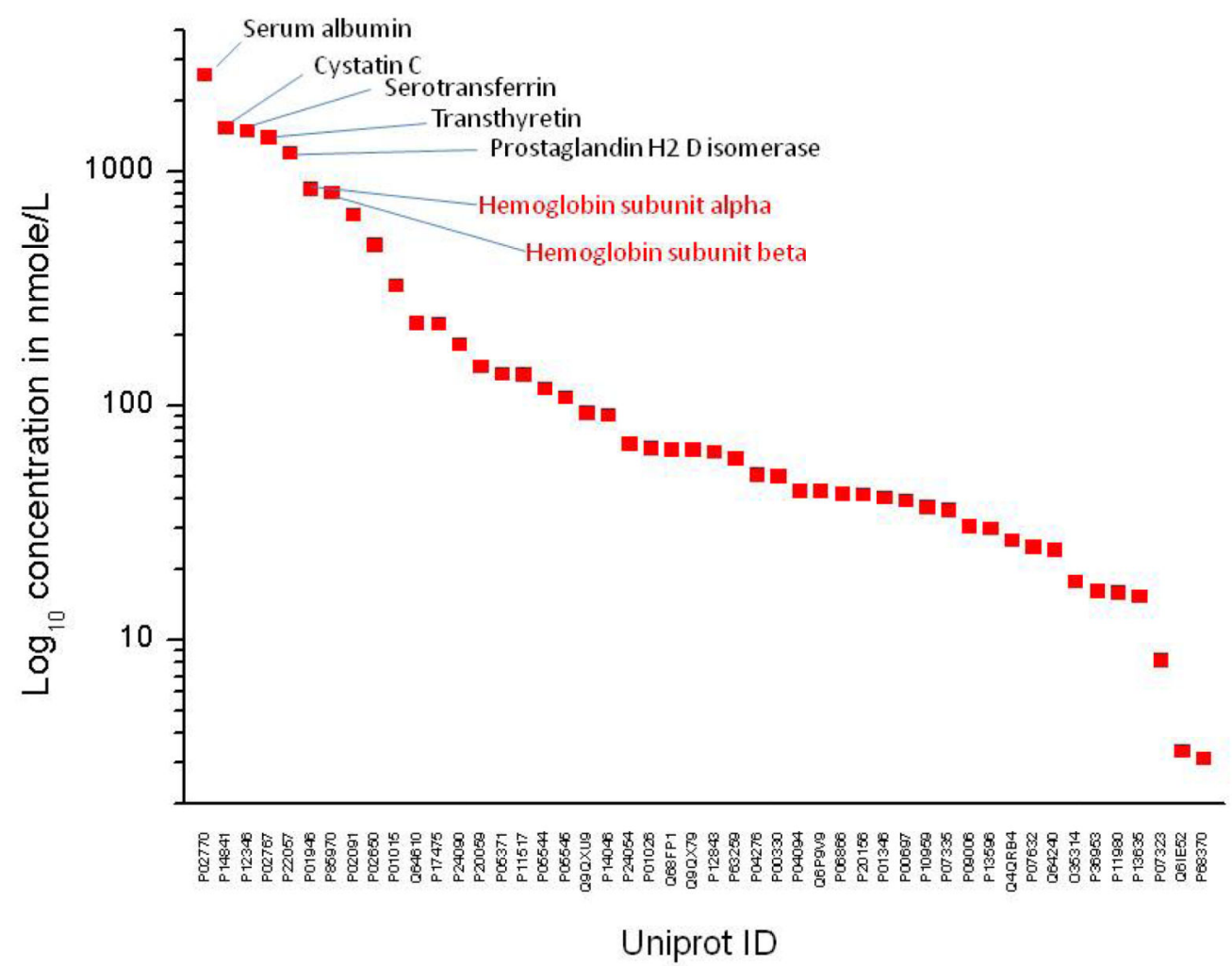

Figure 3.

List of proteins identified in the naïve CSF pooled sample set from control animals $(n=10)$ sorted by concentration. PLGS Score is calculated by the Protein Lynx Global Server (PLGS) software using a Monte Carlo algorithm to analyze all available MS data and is a statistical measure of accuracy of assignment. The data were filtered considering only peptides identified with a high probability (PLGS Score > 150). Alcohol dehydrogenase (50 fmol on column) was used as an internal standard. 


\section{Scores Plot}

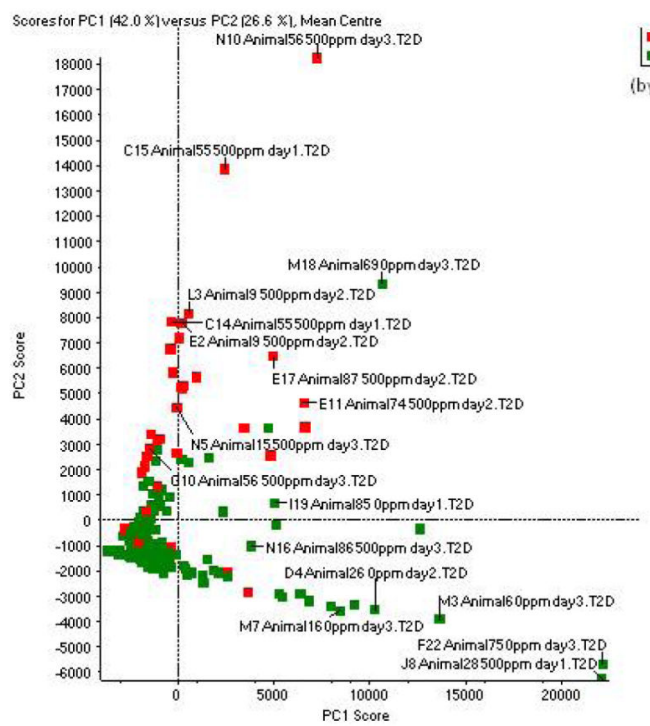

Loading Plot

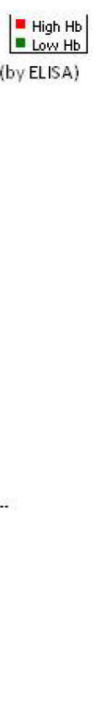

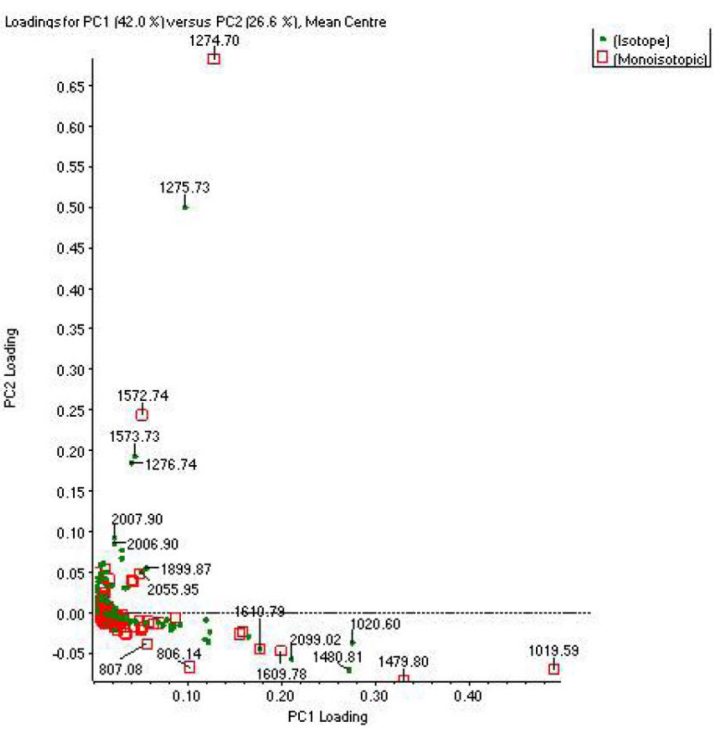

Figure 4.

Principal Component Analysis (PCA) (High Hb versus Low $\mathrm{Hb}$ ). Unsupervised method allows the intersample relationships to be determined and visualized. PC1 $(42.0 \%$ of the variance) does not separate the High $\mathrm{Hb}$-samples from the Low Hb-samples. However, PC2 (26.6\% of the variance) does separate the samples according to blood contamination. 

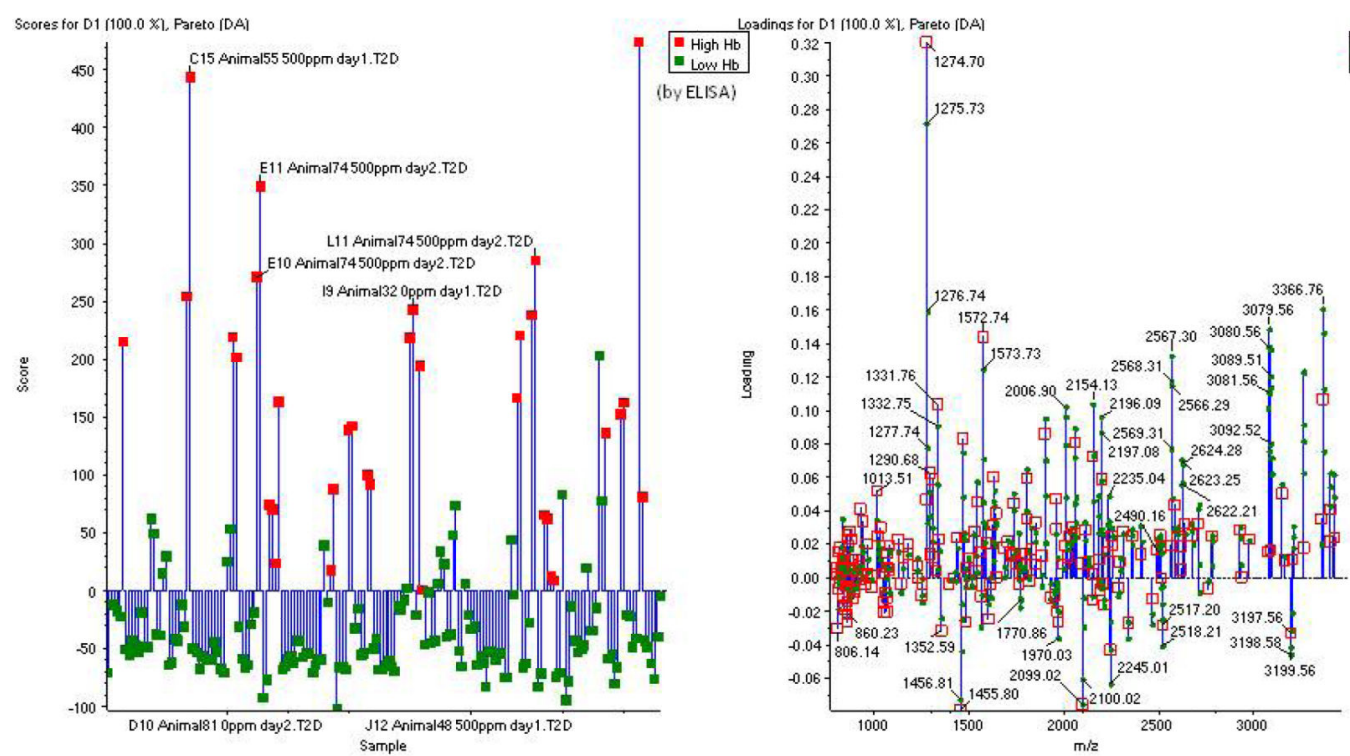

Figure 5.

Discriminant Analysis (PCA-DA) (High Hb versus Low Hb). Prior knowledge of the sample groups (High $\mathrm{Hb}$ versus Low $\mathrm{Hb}$ ) facilitates data interpretation. These samples clearly separate on the basis of the contamination by blood. The majority of the high Hb samples have high positive D1 scores. 


\section{Scores Plot}

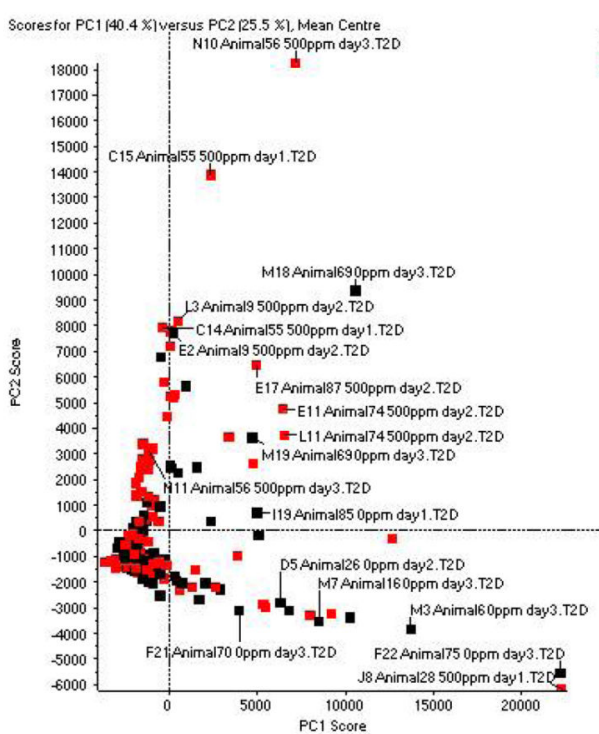

Loading Plot

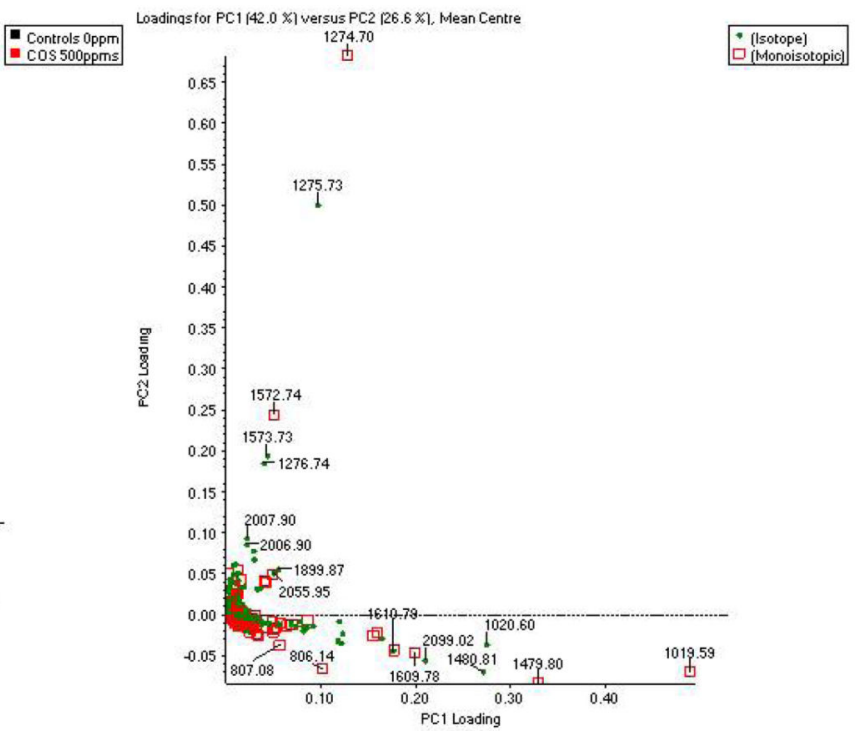

Figure 6.

Principal Component Analysis (PCA) (COS-treated versus Controls). Neither PC1 (40.4\% of the variance) or PC2 (25.5\% of the variance) separates the COS-treated samples from the controls. 


\section{Scores Plot}

\section{Loading Plot}
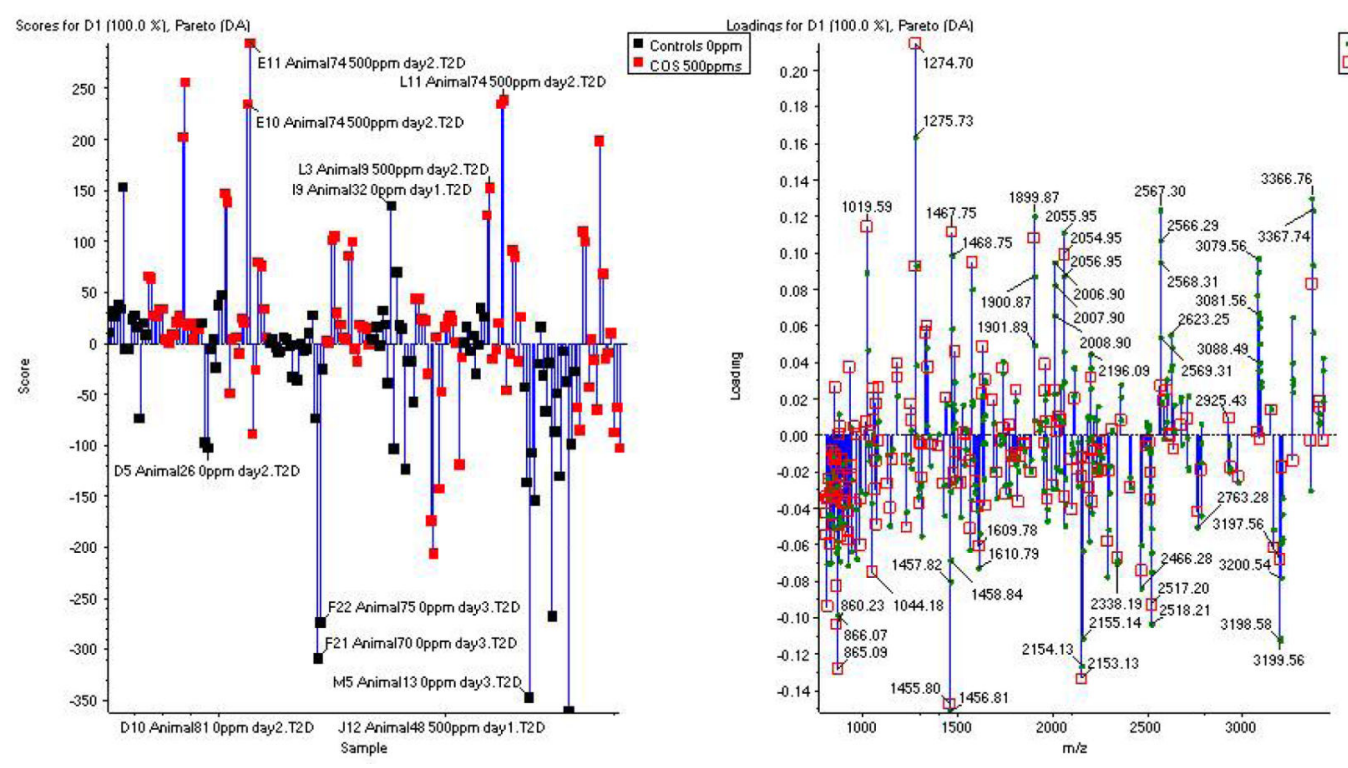

Figure 7.

Discriminant Analysis (PCA-DA) (COS-treated versus Controls). No obvious differences between COS-treated samples and controls could be detected using the supervised method. 


\section{Table 1}

Contamination of CSF samples by blood

\begin{tabular}{lccc}
\hline Type of Sample & \multicolumn{3}{c}{ Number of sample } \\
& "red" $\boldsymbol{a}$ & "clear" $\boldsymbol{a}$ & "Hb free" $\boldsymbol{b}$ \\
\hline 0 ppm day 1 & 6 & 9 & 0 \\
500 ppm day 1 & 5 & 10 & 0 \\
0 ppm day 2 & 10 & 5 & 1 \\
500 ppm day 2 & 7 & 8 & 0 \\
0 ppm day 3 & 4 & 11 & 5 \\
500 ppm day 3 & 7 & 8 & 1 \\
\hline Total number & 39 & 51 & 7 \\
\hline
\end{tabular}

by visual inspection

${ }^{b}$ Sample with hemoglobin levels lower than 1/500,000 dilution of a whole blood sample based on the ELISA assay 


\section{Table 2}

CSF samples analyzed by mass spectrometry

\begin{tabular}{lcc}
\hline Type of Sample & \multicolumn{2}{c}{ Number of sample analyzed } \\
& MALDI-TOF & ESI-Ion Trap \\
\hline 0 ppm day 1 & 9 & 3 \\
500 ppm day 1 & 10 & 3 \\
0 ppm day 2 & 5 & 3 \\
500 ppm day 2 & 8 & 3 \\
0 ppm day 3 & 11 & 3 \\
500 ppm day 3 & 8 & 2 \\
\hline Total number & 51 & 17 \\
\hline
\end{tabular}

Rapid Commun Mass Spectrom. Author manuscript; available in PMC 2015 December 15. 